Introduction: The impaired balance between cell proliferation and cell death, followed the inability to receive the death signals, cells push towards the neoplasia pathway. Fibulin 1 (FBLN1) plays a role as a co-factor in the mechanism of action of a protease such as a disintegrin and metalloproteinase with thrombospondin motifs (ADAMTS-1), which has important roles in angiogenesis, can also act as both tumor suppressor gene (TSG) and an oncogene in the main constituent of the extra-cellular matrix. This preliminary study has investigated the effects of silencing FBLN1 with siRNA on autophagy, proliferation, apoptosis pathways in the MSM cell line.

Material and methods: It was transfected siRNA specific to FBLN1 incubated MSM SPC212 cells, and compared with negative control siRNAs by a real-time polymerase chain reaction. It was determined apoptosis, proliferation, autophagy-related genes in mRNA levels.

Results: It was observed that increased anti-apoptosis genes, such as CASP2, CASP7, DDFA, and BCL2, anti-apoptotic gene, reduced $A P A F 1$, CASP8. Proliferation induced through while increased ADAMTS1, CDH1, CDH6, CLDN7, CSF3, MMP7, MMP13 genes. Autophagy increased via increasing MAP1LC3B, ATG-16L1 genes while decreased via suppressed ULK1, and ATG7 genes by silencing FBLN1 with siRNAs $(p<0.05)$.

Conclusions: Proliferation can be induction with silencing of FBLN1 with siRNA in processing mechanism MSM. It was concluded that FBLN1 could be act as pleiotropic on autophagy, and apoptosis pathways in proliferation processing for MSM. Therefore we think that FBLN1 acts like a TSG. FBLN1 can be considered as a targeted treatment option in advanced stage MSM.

Key words: mesothelioma, fibulin 1, autophagy, proliferation, apoptosis.

Contemp Oncol (Pozn) 2020; 24 (4): 241-246 DOI: https://doi.org/10.5114/wo.2020.102826

\section{A preliminary study: is fibulin 1 a friend or an enemy that needs to be silenced with siRNAs for mesothelioma?}

\author{
Asude Aksoy ${ }^{1}$, Ahmet Tektemur ${ }^{2}$, Elif Melek ${ }^{1}$, Mustafa Kayfeci ${ }^{1}$, \\ Muhammed F. Uslu ${ }^{1}$, Ugurcan Cosar ${ }^{1}$, Ebru Onalan ${ }^{2}$
}

${ }^{1}$ Department of Medical Oncology, Medical Faculty, Firat University, Elazig, Turkey 2Department of Medical Biology and Genetics, Medical Faculty, Firat University, Elazig, Turkey

\section{Introduction}

Highly aggressive MSM treatment options are also very limited, and the median survival does not exceed 1 year with multimodality approaches in MSM. Recently, 18.1 million new cases and 9.6 million deaths were recorded worldwide in 2018 [1, 2].

In MSM etiopathogenesis, cancer-associated fibroblasts are known to direct the immune cells along the process. Fibulin (FBLN), which is one of the building blocks of the extracellular component, has been shown in many cancers to act like a tumor suppressor gene (TSG), and it has been shown in studies that the prognosis was good in tumors where FBLN is overexpressed; FBLN3 even had prognostic utility for MSM. FBLN1, which is a component of the extracellular matrix (ECM), normally prevents the activation of extracellular signal-regulated kinase (ERK) by blocking the phosphorylation of the myosin light chain through fibronectin, and the cell's ability to move remains stable [3-6].

Studies have shown that FBLN1 acts as a cofactor for the enzyme ADAMTS-1. This, a zinc-dependent, matrix metallopeptidase (MMP) enzyme, is a proteoglycan that breaks down adhesion proteins, such as CD44, CD1, $\mathrm{N}$-cadherin, and L1 adhesion molecules, which have been identified in tumor cells. ADAMTS-1 performs these functions through FBLN1, transforming growth factor- $\beta$ (TGF- $\beta$ ), which acts as the cofactors of MMP9. Although ADAMTS-1 is extensively expressed in MSM as in many cancers, it is also involved in MSM invasion, proliferation mechanisms. Studies in MSM human cell lines have shown that MSM progression is reduced with ADAMTS-1 SiRNAs [7-10]. There are still many obscure points in the etiopathogenesis of MSM, unraveling those points will be a beacon for targeted therapies. So far, since the FBLN1 relationship in MSM etiopathogenesis has never been investigated, our objective has to examine the effects of silencing FBLN1, which works like MMP, on proliferation, apoptosis, autophagy in MSM.

\section{Material and methods}

\section{Cell cultures analysis}

RPMI-1640 medium (Cat. No. R0883, Sigma-Aldrich, Germany) containing fetal bovine serum 10\% (Cat. No. F6178, Sigma-Aldrich, USA) was used to grow SPC212 cells (ATCC ${ }^{\circledast}$ CRL-1435 $5^{\mathrm{TM}}$ ). Cells were cultured in an incubator (Nuve, Turkey) with $5 \% \mathrm{CO}_{2}$ and $95 \%$ air at $37^{\circ} \mathrm{C}$. 


\section{SiRNA assay}

SPC212 cells were plated on 6-well cell culture plates and incubated for 24 hours and then transfected with either siRNA specific to FBLN1, (Cat. No. GS2192, Qiagen, USA) or negative control siRNA (Cat. No. 1027280, Qiagen, USA) using HiPerfect ${ }^{\oplus}$ transfection reagent (Cat. No. 301704, Qiagen, Germany) according to the manufacturer's instructions. Using the FBLN1, human gene test (Cat. No. PPH21276A, Qiagen, Germany), quantitative Real Time-Polymerase Chain Reaction (qRT-PCR) was performed to determine whether silencing was occurring. According to the qRT-PCR result, the following calculation method was used to calculate the percentage of siRNA silencing: $\Delta \Delta C T$ mean $=\Delta C T$ related gene (TRPM2) -siRNA $-\Delta C T$ negative control; fold change $=2^{-\Delta \Delta C T}$; percentage of silence $=100 \times(1-$ fold change $)$.

\section{Total RNA isolation from cell culture}

RNA isolation from SPC212 cells was conducted with the Gene Jet RNA Purification kit (Cat. No. K0731, Thermo Scientific, Lithuania) according to the manufacturer's recommended protocol. The RNA pellet resuspended in 10-30 $\mu$ l of nuclease-free water.

\section{Complementary DNA synthesis}

The PCR for complementary DNA (CDNA) synthesis was performed by using a High-Capacity cDNA Reverse Transcription Kit (Cat. No. 4368814, Applied Biosystems, USA), by thermal cycler (Veriti, Applied Biosystems, Singapore) at $25^{\circ} \mathrm{C}$ for $10 \mathrm{~min}, 37^{\circ} \mathrm{C}$ for $120 \mathrm{~min}$ and $85^{\circ} \mathrm{C}$ for $5 \mathrm{~min}$, according to the manufacturer's instructions.

\section{qRT-PCR analysis}

SYBR-green-based on autophagy panel (Cat. No. HATPL-I, Human AUP Primer Library, RealTimePrimers.com), apoptosis panel (Cat. No. HPA-I, Human APO Primer Library, RealTimePrimers.com), proliferation panel (Cat. No. HTIM-I, Human Metastasis, and Invasion Primer Library, RealTimePrimers.com) were used for the gene expression analysis at mRNA level. In Table 1, the genes and their properties assessed using qRT-PCR analysis in SPC212 cells and paraffin-embedded MSM tissue are given. The mixture required for evaluation of TRPM2 and autophagic-apoptotic gene expressions were prepared with iTaq universal SYBR green supermix (Cat. No. 172-5121, Bio-Rad, USA). mRNA expression levels of genes were determined by the qRTPCR system (7500 RT-PCR, Applied Biosystems, Singapore). In the study glyceraldehyde 3-phosphate dehydrogenase (Cat. No. QT00079247, Qiagen, USA) was used as a control gene (housekeeping). PCR measurements were repeated three times and these measurements were obtained. At the end of the qRT-PCR analysis, 2- $\Delta \Delta C T$ method was used to calculate the differences in gene expression.

\section{Statistical analysis}

All descriptive and inferential statistical analyses were performed with IBM Statistical Package for the Social Sciences (SPSS) version 22.0 software (Chicago, IL, USA). De- pending on data distribution, the Mann-Whitney test or ANOVA was conducted for group comparisons. Pearson correlation coefficient was determined to study relations between variables. The qRT-PCR data were analyzed by using the $\Delta \Delta$ Ct module at the QiagenGeneGlobe Data Analysis Center portal: http://www.qiagen.com/us/shop/genesand-pathways/data-analysis-center-overview-page/. The qRT-PCR module transforms the threshold cycle (Ct) values to calculate results for gene expression. The efficiency of all the primers used in over $90 \% . p<0.05$ was considered as significant.

\section{Results}

After the siRNA transfection FBLN1, the mRNA expression level was detected by qRT-PCR. FBLN1 mRNA expression levels, in siRNA groups, demonstrated a significant decrease against to control group $(p=0.0084)$. The percentage of siRNA silencing was calculated as $81.3 \%$ by using the gene silencing calculation method in applied biosystems (Fig. 1).

The outcome of the siRNA-mediated knockdown of FBLN1, apoptosis up-regulated by increasing the anti-apoptotic gene, BCL2, and AFAP1, CASP8 genes, although down-regulated by increasing the proapoptotic genes CASP2, CASP7, DFFA. The genes of proliferation such as ADAMTS1, CDH1, CDH6, CLND7, CSF3, MMP13, MMP7, and $A T G 16 L$ increased. Autophagy decreased by decreasing ULK, ATG7 genes but increased by increasing the MAP1LC3B gene $(p<0.05)$ (Table 1).

\section{Discussion}

Many cancer cells have developed various mechanisms to avoid cell death by increasing the levels of anti-apoptotic molecules or by inactivating pro-apoptotic cell death components. In carcinogenesis, this process is reflected as a problem in any steps of the apoptotic pathways [1]. The adaptation of tumor cells that overcome this step to the microenvironment is the main target of tumor growth. Autophagy plays a catabolic role in this adaptation, acts as a TSG. Often, a malignant event occurs by the inhibition of autophagy mechanisms, after which metastatic pathways are activated [11].

In recent years, small interfering RNA (siRNAs) that stop the expression of genes and prevent the progression and formation of many diseases are quite popular for the treatment of diseases. siRNAs serve as guide RNAs for proper micro RNA (miRNA) degradation. It has been shown that they can be used as a new potential target in cancer therapy [12].

For the treatment of MSM, siRNAs have been used in many in the vitro study setting. It has been proven that silencing MSM genes with siRNAs can be novel targeted therapies, both alone and in combination with chemotherapy agents [13].

Out of the 4 different splice forms, FBLN1 C and D forms are shown to be the most abundant in cancer tissues. With FBLN1 C form in the oncogenic pattern and FBLN1 D form in the TSG pattern, FBLN1 is in a dual format. FBLN1 likely put on a pleiotropic pattern due to this dual property of 


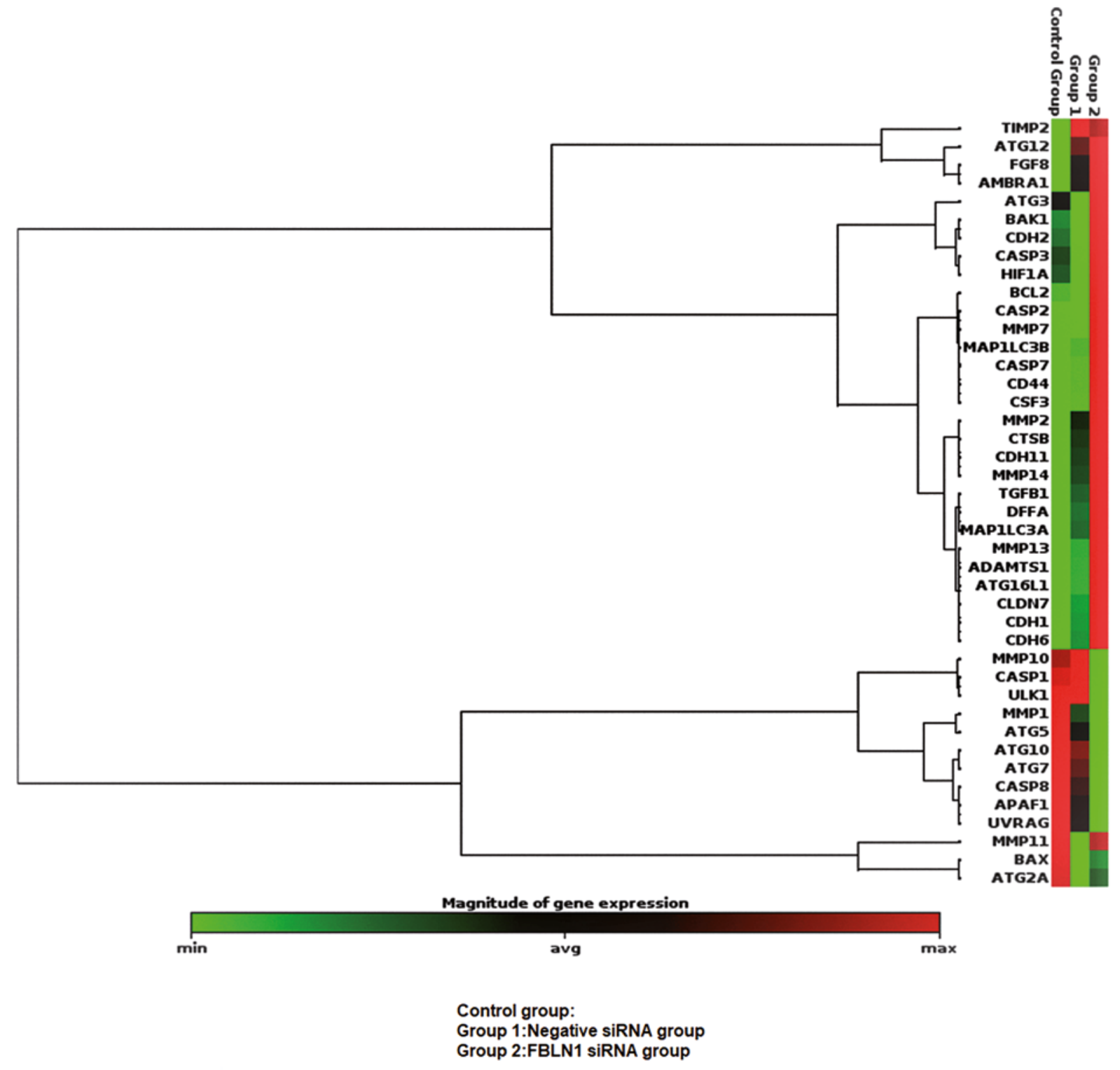

Fig. 1. Heat map of the effects on gene expression after the transfection FBLN1 siRNA in mesothelioma cell line

The MRNA expression obtained using qPCR for the targets selected from the genes in Table 1 is shown as a graph of the profile of the heat map. Genes clustered according to their expression patterns. The red color on the heat map indicates genes that are highly expressed compared to control; green color, genes that are expressed at low level; whereas black color represents genes that are equal to the control.

its. A mutation in the FBLN1 gene locus can affect many properties, such as the pleiotropic phenomenon. The ratio FBLN1C: FBLN-1D shifting to the FBLN1D side with estradiol containing treatments. It has been demonstrated in studies, that a shift towards FBLN1C plays a major role during carcinogenesis stages. It has been shown that hormonal treatments are given where $F B L N 1$ is overexpressed inhibits the metastatic process by blocking the ERK pathway by binding to fibronectin $[8,14]$.

During carcinogenesis, the balance between cell proliferation, and cell death, is impaired. The death signals that will put cells into the apoptosis pathway cannot be received. Many cancer cells can avoid apoptosis by increasing the levels of anti-apoptotic molecules or by inactivating pro-apoptotic cell death components. Escape from apoptosis, promoting carcinogenesis occurs via path- ways such as impairment of balance in $\mathrm{BCL}-2$ proteins, decreased caspase (CASP) function, impaired receptor signaling pathway, increased expression of apoptosis inhibitor family molecules, p53 defect mutations $[15,16]$.

In this study, we can think that MSM uses an intrinsic apoptotic pathway with the down-regulation of APAF1 by silencing FBLN1 with siRNA in MSM cell lines. After the FBLN1 SiRNA transfection as a result of the up-regulation of $B C L 2$, located on the outer membrane of the mitochondria, the balance is disturbed among the members of the $B C L-2$ family ( $\mathrm{BCl}-\mathrm{Xl}, \mathrm{Mcl}-1)$. It is observed that apoptosis isn't induced because with increased BCL2, APAF1 to create the apoptosome, and also CASP9/3 signal cascade is not affected in this study. Studies have shown that CASP2 protein has non-apoptotic functions and can even function in the presence of a p53 [17]. Many oncoprint mutations and deep 
Table 1. mRNA fold change and $p$-values in the MSM cell line compared to the control group after FBLN1 siRNA transfection

\begin{tabular}{|c|c|c|c|c|c|}
\hline \multirow[t]{2}{*}{ Symbol } & \multirow[t]{2}{*}{ Name } & \multicolumn{2}{|c|}{ Negative control } & \multicolumn{2}{|c|}{ FBLN1 SIRNA } \\
\hline & & p-value & $\begin{array}{c}\text { mRNA } \\
\text { fold change }\end{array}$ & $p$-value & $\begin{array}{c}\text { mRNA } \\
\text { fold change }\end{array}$ \\
\hline GAPDH & Glyceraldehyde-3-phosphate dehydrogenase & 1 & 0 & 1 & 0 \\
\hline APAF1 & Apoptotic peptidase activating factor 1 & 0.79 & 0.37376 & 0.4698 & 0.049464 \\
\hline BAK1 & BCL2-antagonist/killer 1 & 0.9593 & 0.85223 & 1.1567 & 0.585246 \\
\hline BAX & BCL2-associated $X$ protein & 0.7423 & 0.280302 & 0.8011 & 0.410412 \\
\hline $\mathrm{BCL} 2$ & B-cell CLL/lymphoma 2 & 0.8546 & 0.536878 & 3.4822 & 0.014865 \\
\hline CASP1 & Caspase 1 , apoptosis-related cysteine peptidase & 1.0353 & 0.906699 & 0.115 & 0.007203 \\
\hline CASP2 & Caspase 2, apoptosis-related cysteine peptidase & 0.9593 & 0.85223 & 34.0598 & 0.004306 \\
\hline CASP3 & Caspase 3, apoptosis-related cysteine peptidase & 0.8293 & 0.466433 & 1.3379 & 0.301517 \\
\hline CASP7 & Caspase 7, apoptosis-related cysteine peptidase & 1.7532 & 0.085083 & 22.6274 & 0.004565 \\
\hline CASP8 & Caspase 8, apoptosis-related cysteine peptidase & 0.7792 & 0.350619 & 0.3392 & 0.022406 \\
\hline DFFA & DNA fragmentation factor, $45 \mathrm{kDa}$, alpha polypeptide & 1.2658 & 0.383415 & 2.1435 & 0.045288 \\
\hline ADAMTS1 & ADAM metallopeptidase with thrombospondin type 1 motif,1 & 1.3566 & 0.273954 & 4.3169 & 0.010946 \\
\hline CD44 & CD44 molecule (Indian blood group) & 1.0281 & 0.928655 & 1.879 & 0.070592 \\
\hline $\mathrm{CDH} 1$ & Cadherin 1, type 1, E-cadherin (epithelial) & 1.257 & 0.396375 & 2.5315 & 0.028459 \\
\hline $\mathrm{CDH} 2$ & Cadherin 2, type 1, N-cadherin (neuronal) & 0.8409 & 0.496373 & 1.4948 & 0.180539 \\
\hline $\mathrm{CDH} 6$ & Cadherin 6, type 2, K-cadherin (fetal kidney) & 1.6021 & 0.125128 & 4.4076 & 0.010672 \\
\hline $\mathrm{CDH} 11$ & Cadherin 11, type 2, OB-cadherin (osteoblast) & 1.3013 & 0.335359 & 1.879 & 0.070592 \\
\hline CLDN7 & Claudin 7 & 1.6472 & 0.110732 & 5.2054 & 0.008915 \\
\hline CSF3 & Colony stimulating factor 3 (granulocyte) & 1.1019 & 0.716013 & 4.3772 & 0.010762 \\
\hline CTSB & Cathepsin B & 1.0425 & 0.884836 & 1.1173 & 0.676429 \\
\hline FGF8 & Fibroblast growth factor 8 (androgen-induced) & 1.4142 & 0.223932 & 1.7532 & 0.091797 \\
\hline HIF1A & Hypoxia inducible factor 1 , alpha subunit & 0.9138 & 0.707769 & 1.2142 & 0.472539 \\
\hline MMP1 & Matrix metallopeptidase 1 (interstitial collagenase) & 0.8123 & 0.424436 & 0.717 & 0.247844 \\
\hline MMP2 & Matrix metallopeptidase 2 (gelatinase A) & 1.2142 & 0.467054 & 1.5369 & 0.159477 \\
\hline MMP7 & Matrix metallopeptidase 7 (matrilysin, uterine) & 1.2226 & 0.452126 & 174.8532 & 0.003936 \\
\hline MMP13 & Matrix metallopeptidase 13 (collagenase 3) & 1.2142 & 0.467054 & 2.8284 & 0.021993 \\
\hline MMP14 & Matrix metallopeptidase 14 (membrane-inserted) & 1.3195 & 0.313522 & 1.9862 & 0.058051 \\
\hline TGFB1 & Transforming growth factor, beta 1 & 1.2142 & 0.467054 & 1.7901 & 0.084661 \\
\hline TIMP2 & TIMP metallopeptidase inhibitor 2 & 1.3566 & 0.273954 & 1.3195 & 0.321744 \\
\hline AMBRA1 & Autophagy/beclin-1 regulator 1 & 1.5263 & 0.15604 & 1.9453 & 0.062375 \\
\hline ATG5 & ATG5 autophagy related 5 homolog (S. cerevisiae) & 0.8351 & 0.481208 & 0.6926 & 0.212182 \\
\hline ATG7 & ATG7 autophagy related 7 homolog (S. cerevisiae) & 0.7955 & 0.385871 & 0.2017 & 0.010764 \\
\hline ATG12 & ATG12 autophagy related 12 homolog (S. cerevisiae) & 1.2924 & 0.346816 & 1.3851 & 0.256392 \\
\hline ATG10 & ATG10 autophagy related 10 homolog (S. cerevisiae) & 0.9266 & 0.747767 & 0.6156 & 0.12827 \\
\hline ATG16L1 & ATG16 autophagy related 16-like 1 (S. cerevisiae) & 1.2746 & 0.370839 & 3.6301 & 0.013914 \\
\hline MAP1LC3A & Microtubule-associated protein 1 light chain 3 alpha & 1.2142 & 0.467054 & 1.8532 & 0.074279 \\
\hline MAP1LC3B & Microtubule-associated protein 1 light chain 3 beta & 1.0644 & 0.820037 & 2.0994 & 0.048348 \\
\hline ULK1 & Unc-51-like kinase 1 (C. elegans) & 0.9931 & 0.961064 & 0.1142 & 0.007178 \\
\hline UVRAG & UV radiation resistance associated gene & 0.8351 & 0.481208 & 0.5905 & 0.108666 \\
\hline ATG2A & ATG2 autophagy related 2 homolog A (S. cerevisiae) & 0.8293 & 0.466433 & 0.8827 & 0.626572 \\
\hline ATG3 & ATG3 autophagy related 3 homolog (S. cerevisiae) & 0.7738 & 0.339578 & 1.2397 & 0.429877 \\
\hline
\end{tabular}

deletions have been detected in 74 pleural MSM tumors in the cancer genome atlas. MSM is largely associated with the result of a loss of function of TSGs. There are $46 \%$ cyclin-dependent kinase inhibitor 2A, 28\% BRCA1 associated protein-1, 30\% neurofibromatosis tip 2, 6\% large tumor sup- pressor homolog 2, 15\% p53 mutations, although there is no change in tumor suppressor gene (TSG) that are common in $25 \%$ of tumors [18]. It's suggested that there are undefined tumor suppressor pathways in MSM. We can make an indirect deduction that p53 mutations are seen more in 
MSM because CASP2, one of the initiator CASPS, has been up-regulated by FBLN1 with SiRNAS. CASP2 has been shown to function as a TSG in an animal study but no human study [19]. In this study, it is considered that CASP2 decreased as a TSG in MSM due to it is down-regulated after transfection FBLN1 SiRNA. CASP7, an effector of CASP, was down-regulated by silencing FBLN1 with siRNAs, contributed to the inhibition of apoptosis. DFFA is, the substrate of CASP3, and it also triggers DNA fragmentation during apoptosis, has been down-regulated by silencing FBLN1 with siRNAs. However, studies have shown that there is an inverse correlation between poor prognostic features, and DFFA expression in advanced stage esophageal cancer cases [20]. However, it was observed that there is an inverse relationship between the increased levels of CASP3 and prognosis in some cancers, CASP3 down-regulation was not observed in this study [21].

The invasion mechanism stopped by up-regulating $\mathrm{CDH} 1$, which was thought to be predominant in the etiopathogenesis of MSM, by FBLN1 siRNA [22]. CDH6 has been shown to acts like a TSG, and also the relationships between its dysregulation and cholangiocarcinomas have been pointed out. Positive results of $\mathrm{CDH} 6$ based on the targeted treatment of various tumors have also been shown in studies. Also in our study, it was observed that $\mathrm{CDH} 6$ up-regulation by FBLN1 siRNAs protected to invasion [23].

Studies have shown that claudin 7 (CLDN7) is immune expressed, not so potently, in $43 \%$ of MSM. CLDN7, one of the epithelial cell adhesion molecules, has been shown to play a role in cell-to-cell adhesion, proliferation, metastasis. There are different opinions in the literature with CLDN7 [24]. Although overexpression of CLDN7 in the ovarian cells has increased progression, the studies in breast cancers have also shown increased progression with loss of CLDN7 expression $[25,26]$. It is observed that invasion can be prevented by the upregulation of CLDN7 as a result of the silencing of FBLN1 siRNAs in MSM cell lines. It means that invasion can be fought. Although no relationship between CLDN7 and survival was demonstrated in the literature, it should be supported by further studies on this subject [24].

Under normal conditions, the cross-talking nature between the T-cells, tumor cells, and the granulocytic series cells in MSM are not fully understood. Granulocyte-macrophage colony-stimulating factors increase the motility, maturation of dendritic cells as well as increase cytotoxic T cell function activation. In a study, it has been shown that with colony-stimulating factor 3 (CSF3) in MSM cells, free reactive oxygen production can be increased, which suppresses the function, some T cells [3, 4]. In our study, it was found that CFS3 down-regulated by FBLN1 silencing with siRNAs, thereby acting to prevent proliferation by indirectly contributing to the function of cytotoxic T cells in proliferation biology.

It has been demonstrated with studies that MMP, blocking of ECM, plays a role in MSM etiopathogenesis through mitogen-activated protein kinase / ERK and c-Jun N-terminal kinase pathways $[3,4,27]$. With the current study, the up-regulation of MMP7 in MSM cell lines with siRNA FBLN1s has been shown to prevent the spread of invasion. Thus, it can be suggested that low MMP7 levels play a role in the etiopathogenesis of MSM.
It was observed that MMP13 helps to reduce metastatic potential up-regulated by FBLN1 siRNAs, as it contributes to a tyrosine kinase-like phosphorylation cascade in the ECM [27]. From here we can assume that phosphatidylinositol 3-kinase/ protein kinase B/ mammalian target of rapamycin pathway is mutated in MSM. In contrast, in some cancers, an increase of MMP 13 has been associated with poor prognosis [29].

Autophagy is a pivotal route to maintain the vital functions of the cell. While basal autophagy functions as a TS mechanism during tumorigenesis, some cancers can also survive with exaggerated autophagy. During autophagosome formation, ATG5, and ATG12 are linked by a ubiquitin-like binding system, ATG5-ATG12/ATG16 complex is formed. In some cancers functions of autophagy have not been fully understood, the development of hepatocellular cancer is observed after the defects in ATG5-7 genes [30].

Autophagy-related protein microtubule-associated protein 1A/1B-light chain 3 (LC3) and ATG16L1, which is one of the key proteins of autophagy and which provides a basis for autophagosome formation after cross-talking with many genes, has contributed to the augmentation of autophagy by being up-regulated by FBLN1 siRNAs in our study.

Our study has several limitations. First, we used only one MSM cell line in this study. Wish, we could have been used more different MSM cell lines, it would be able to compare cell biological properties such as in vitro cell growth, apoptosis, motility, and proliferation of MSM. Second, we didn't conduct the presence of alternative splice variants of these genes and genetic alterations that occur throughout the passage of cell lines. If we could have carried out the analysis for alternative splice variants of those genes and genetic alterations, we would be able to discuss more accurate and more objective results regarding in proliferation processing mechanism in MSM. Thirdly, this study is preliminary featured. It has not done apoptosis, autophagy, cell proliferation analyses by western blot due to this study is preliminary featured. This part of the study will be considered as the main purpose of another study.

\section{Conclusions}

The fact that in MSM cell lines, rather than apoptosis genes, some of the invasion and autophagy genes have been in vitro silenced with FBLN1 siRNAs has shown that FBLN1 acts as a TSG in the early stages of carcinogenesis, and the invasion of MSM is mainly through MMP7. Whether it is an apoptotic, invasion, or autophagy pathway, many genes repeatedly determine the directions of their anabolic functions through cross-talks between each other. It is observed that FBLN1 can act as a TSG in the early stages and by silencing FBLN1 with siRNAs, tumor metastases increase and autophagy partially increases. With more extensive studies, both in vivo and in vitro, new targeted therapy options might be created, with up-regulated of FBLN1, along with the agents acting via different mechanisms of action in the metastatic process of MSM. 


\section{Acknowledgements}

This study has received financial support from the Scientific Research Project of Firat University (no: TF. 15. 47/ 2015).

The authors declare no conflict of interest.

\section{References}

1. Green DR, Galluzzi L, Kroemer G. Metabolic control of cell death. Science 2014; 345: 1250256.

2. Ferlay J, Colombet M, Soerjomataram I, Mathers C, Parkin DM, Piñeros M, Znaor A, Bray F. Estimating the global cancer incidence and mortality in 2018: GLOBOCAN sources and methods. Int I Cancer 2019; 15: 1941-1953.

3. Yang H, Testa JR, Carbone M. Mesothelioma epidemiology, carcinogenesis, and pathogenesis. Curr Treat Options Onco 2008; 19: 147-157.

4. Yilmaz M, Christofori G. EMT, the cytoskeleton, and cancer cell invasion. Cancer Metastasis Rev 2009; 28: 15-33.

5. Spence SG, Argraves WS, Walters L, Hungerford JE, Little CD. Fibulin is localized at sites of epithelial-mesenchymal transitions in the early avian embryo. Dev Biol 1992; 151: 473-484.

6. Tran H, Van Dusen WJ, Argraves WS. The Self-association and Fibronectin-binding Sites of Fibulin-1 Map to Calcium-binding Epidermal Growth Factor-like Domains. The Journal of Biological Chemistry 1997; 272: 22600-22606.

7. Lee NV, Rodriguez-Manzaneque JC, Thai SN, Twal WO, Luque A, Lyons KM, Argraves WS, Iruela-Arispe ML. Fibulin-1 acts as a cofac tor for the matrix metalloprotease ADAMTS-1. J Biol Chem 2005; 280: 34796-34804

8. Gallagher WM, Currid CA. Fibulins and cancer: friend or foe? Whelan LC. Trends Mol Med 2005; 11: 336-40.

9. Sépult C, Bellefroid M, Rocks N, et al. ADAM10 mediates malignant pleural mesothelioma invasiveness. Oncogene 2019; 38: 3521-34.

10. Qing J, Maher VM, Tran H, Scott AW, Dunstan WR, McCormick JJ. Suppression of anchorage-independent growth and matrigel invasion and delayed tumor formation by elevated expression of fibulin-1D in human fibrosarcoma-derived cell lines. Oncogene 1997; 15: 2159-2168.

11. Liu H, He Z, Simon H. Protective role of autophagy and autophagy-related protein 5 in early tumorigenesis. J Mol Med 2015; 93: 159-164.

12. Drakaki A, Iliopoulos D. MicroRNA gene networks in oncogenesis. Curr Genomics 2009; 10: 35-41.

13. Ombretta M, Justin S, Ylenia L, et al. MSLN Gene Silencing Has an Anti-Malignant Effect on Cell Lines Overexpressing Mesothelin Deriving from Malignant Pleural Mesothelioma. PLoS One 2014; 2: 0180317.

14. Paaby AB, Rockman MV. The many faces of pleiotropy. Trends Genet 2013; 29: 66-73.

15. Frenzel A, Grespi F, Chmelewskij W, Villunger A. Bcl2 family proteins in carcinogenesis and the treatment of cancer. Apoptosis 2009; 14: 584-96.

16. Sidi S, Sanda T, Kennedy RD, et al. Chk1 Suppresses a caspase-2 Apoptotic Response to DNA Damage That Bypasses p53, Bcl-2, and caspase-3. Cell 2008; 133: 864 -77.

17. Boice A, Bouchier-Hayes L. Targeting Apoptotic Caspases in Cancer. Biochim Biophys Acta Mol Cell Res. 2020; 1867: 118688.

18. Hmeljak J, Sanchez-Vega F, Hoadley KA, et al. Integrative Molecular Characterization of Malignant Pleural Mesothelioma. E Cancer Discov 2018; 8; 1548-65.

19. Holleman A, den Boer ML, Kazemier KM, Beverloo HB, von Bergh AR, Janka-Schaub GE, Pieters R. Decreased PARP and procaspase-2 protein levels are associated with cellular drug resistance in childhood acute lymphoblastic leukemia. Blood 2005; 106: 1817-23.

20. Konishi S, Ishiguro H, Shibata Y, et al. Decreased expression of DFF45 /ICAD is correlated with a poor prognosis in patients with esophageal carcinoma. Cancer 2002; 95: 2473-78.
21. Hu O, Peng J, Liu W, He X, Cui L, Chen X, Yang M, Liu H, Liu S, Wang H. Elevated cleaved caspase- 3 is associated with shortened overall survival in several cancer types. Int. J. Clin. Exp. Pathol. 7: 50575070. (2014)

22. Abutaily AS, Collins JE, Roche WR. Cadherins, Catenins and APC in Pleural Malignant Mesothelioma. J Pathol 2003; 201: 355-62.

23. Goeppert B, Ernst C, Baer C, et al. Cadherin- 6 is a putative tumor suppressor and target of epigenetically dysregulated miR-429 in cholangiocarcinoma. Epigenetics 2016; 11: 780-90.

24. Soini Y, Kinnula V, Kahlos K, Pääkkö P. Claudins in Differential Diagnosis Between Mesothelioma and Metastatic Adenocarcinoma of the Pleura. J Clin Pathol 2006; 59: 250-54.

25. Dahiya N, Becker KG, Wood WH, Zhang Y, Morin PJ. Claudin-7 Is Frequently Overexpressed in Ovarian Cancer and Promotes Invasion. PLoS One 20116; 7: e22119.

26. Kominsky SL, Argani P, Korz D, et al. Loss of the tight junction protein claudin-7 correlates with histological grade in both ductal carcinoma in situ and invasive ductal carcinoma of the breast. Oncogene 2003; 22: 2021-33.

27. Alì G, Borrelli N, Riccardo G, et al. Differential expression of extracellular matrix constituents and cell adhesion molecules between malignant pleural mesothelioma and mesothelial hyperplasia. J Thorac Oncol 2013; 8: 1389-1395.

28. Bordoli MR, Yum J, Breitkopf SB, et al. A secreted tyrosine kinase acts in the extracellular environment. Cell 2014; 158: 1033-1044.

29. Zhang B, Cao X, Liu Y, et al. Tumor-derived matrix metalloproteinase-13 (MMP-13) correlates with poor prognosis of invasive breast cancer. BMC Cancer 2008; 8: 83.

30. Chang Y, Lin J, Tsung A. Manipulation of autophagy by MIR375 generates antitumor effects in liver cancer. Autophagy 2012; 8: 1833-1834.

\section{Address for correspondence}

\section{Asude Aksoy}

Department of Medical Oncology

Medical Faculty

Firat University

+903-23100 Elazig, Turkey

e-mail: asudeaksoy@hotmail.com

Submitted: 30.08 .2020

Accepted: 22.10 .2020 